\title{
ON THE DUALS OF BINARY HYPER-KLOOSTERMAN CODES
}

\author{
MARKO MOISIO*
}

\begin{abstract}
Binary hyper-Kloosterman codes $C(r, m)$ of length $\left(2^{r}-1\right)^{m-1}$ are a quasi-cyclic generalization of the dual of the Melas code of length $2^{r}-1$. In this note the duals $C^{\perp}(r, m)$ i.e. a generalization of the Melas code $C^{\perp}(r, 2)$ itself are studied. In particular, the minimum distance of $C^{\perp}(r, m)$ for all $r, m \geq 2$, the weight distribution of $C(2, m)$ and $C^{\perp}(2, m)$ for all $m \geq 2$, and the weight distribution of $C(r, 3)$ and $C^{\perp}(r, 3)$ for all $r \geq 2$ is obtained.
\end{abstract}

Key words. Exponential sum, Fermat curve, hyper-Kloosterman code, Kloosterman sum, Melas code, Pless power moments, Weight distribution

AMS subject classifications. 11T23, 11T71

1. Introduction. Let $r, m \geq 2$ be integers and let $q=2^{r}$. Let $\mathbb{F}:=\mathbb{F}_{q}$ denote the finite field of $q$ elements and let $\mathbb{F}^{*}:=\mathbb{F} \backslash\{0\}$. For $\mathbf{a}:=\left(a_{1}, \ldots, a_{m}\right) \in \mathbb{F}^{m}$ we define a rational function in $m-1$ variables:

$$
f_{\mathbf{a}}(\mathbf{X}):=a_{1} X_{1}+\cdots+a_{m-1} X_{m-1}+\frac{a_{m}}{X_{1} \cdots X_{m-1}} .
$$

Let $\mathbf{x}_{1}, \ldots, \mathbf{x}_{n}$ be a fixed ordering of the elements of $\left(\mathbb{F}^{*}\right)^{m-1}$.

In [3] the following linear code $C(r, m)$ was introduced and it was called a hyperKloosterman code:

$$
C(r, m)=\left\{c(\mathbf{a}):=\left(\operatorname{tr}\left(f_{\mathbf{a}}\left(\mathbf{x}_{1}\right)\right), \ldots, \operatorname{tr}\left(f_{\mathbf{a}}\left(\mathbf{x}_{n}\right)\right)\right) \mid \mathbf{a} \in \mathbb{F}^{m}\right\}
$$

here $t r$ is the trace function from $\mathbb{F}$ onto $\mathbb{F}_{2}$. These codes are a quasi-cyclic generalization of the Kloosterman code, i.e. the dual of the Melas code, of length $2^{r}-1$. For the proof of the quasi-cyclicity we refer to [4, Theorem 4.2].

In this note we are interested in the duals $C^{\perp}(r, m)$ which are a generalization of the Melas code $C^{\perp}(r, 2)(r>2)$. We shall show that the minimum distance of $C^{\perp}(r, m)$ is three if $m>2$, and give the weight distribution of $C(2, m)$ and $C^{\perp}(2, m)$ for all $m \geq 2$, and the weight distribution of $C(r, 3)$ and $C^{\perp}(r, 3)$ for all $r \geq 2$. We remark that the weight distributions of $C(r, 2)$ and $C^{\perp}(r, 2)(r>2)$ were obtained in [5] and in [14], respectively.

The rest of this paper is organized as follows. In Section 2 we first consider some simple basic properties of hyper-Kloosterman codes. Next, the weight distribution of $C(r, m)$ is given in terms of certain monomial exponential sums (Theorem 2.5), and then, a recursion formula for the weight distribution of $C^{\perp}(r, m)$ involving the moments $M_{j}$ of those exponential sums is obtained by using the Pless power moment identity (Theorem 2.8).

In Section 3 we first connect the moments $M_{j}$ to a Fermat curve $\mathcal{X}$, and then obtain the number of weight three codewords in $C^{\perp}(r, m)$ in terms of the number of rational points on $\mathcal{X}$ (Theorem 3.2). Finally, we determine the minimum distance of $C^{\perp}(r, m)$ by either using our explicit knowledge of the number of rational points on $\mathcal{X}$ or by estimating that number by either the Hasse-Weil bound or a bound which we shall derive by using Deligne's bound on hyper-Kloosterman sums (Theorem 3.7).

*Department of Mathematics and Statistics, Faculty of Technology, University of Vaasa, PO. Box 700, FIN-65101, Finland(mamo@uwasa.fi). 
In a few cases we are forced to calculate the number of rational points numerically since neither of those bounds is then strong enough.

In Sections 4 and 5 we determine the weight distribution of the codes $C(r, m)$ and $C^{\perp}(r, m)$ in the special cases $r=2, m>2$, and $r>2, m=3$, respectively. In the latter case a relation between one and two dimensional Kloosterman sums from [1] is used, and then, the weight distribution of $C(r, 3)$ is obtained by using results on the distribution of values of Kloosterman sums obtained in [5] (Theorem 5.3). Finally, the weight distribution of $C^{\perp}(r, 3)$ is obtained in terms of even moments of Kloosterman sums calculated in [10] by using result from [14]. Especially, explicit formulae for the number of codewords of weights from three to five is given (Theorem 5.5).

2. On the weight distribution of $C(r, m)$ and $C^{\perp}(r, m)$. Let $\chi$ be the canonical additive character of $\mathbb{F}$. Let

$$
k_{m-1}(\mathbf{a})=\sum_{x_{1}, \ldots, x_{m-1} \in \mathbb{F}^{*}} \chi\left(a_{1} x_{1}+\ldots+a_{m-1} x_{m-1}+a_{m}\left(x_{1} \cdots x_{m-1}\right)^{-1}\right),
$$

be an $(m-1)$-dimensional Kloosterman sum. If $\mathbf{a}=(1,1, \ldots, 1, a)$ with $a \neq 0$ we use the notation

$$
k_{m-1}(a):=k_{m-1}(\mathbf{a})
$$

Let $v$ be the number of zero-components of $\mathbf{a}$. Assume $v>0$. If $a_{m}=0$ then, by the orthogonality of characters, we get

$$
k_{m-1}(\mathbf{a})=\sum_{x_{1}, \ldots, x_{m-1} \in \mathbb{F}^{*}} \chi\left(a_{1} x_{1}+\cdots+a_{m-1} x_{m-1}\right)=(-1)^{m-v}(q-1)^{v-1} .
$$

If $a_{m} \neq 0$ and e.g. $a_{1}=0$ then, by the substitution $y=x_{1}^{-1}$, we obtain

$$
\begin{aligned}
k_{m-1}(\mathbf{a}) & =\sum_{x_{2}, \ldots, x_{m-1} \in \mathbb{F}^{*}} \chi\left(a_{2} x_{2}+\cdots+a_{m-1} x_{m-1}\right) \sum_{y \in \mathbb{F}^{*}} \chi\left(\frac{a_{m}}{x_{2} \cdots x_{m-1}} y\right) \\
& =-\sum_{x_{2}, \ldots, x_{m-1} \in \mathbb{F}^{*}} \chi\left(a_{2} x_{2}+\cdots+a_{m-1} x_{m-1}\right) \\
& =-(-1)^{m-2-(v-1)}(q-1)^{v-1} .
\end{aligned}
$$

Hence we have

LEMMA 2.1. If exactly $v>0$ of the components of $\mathbf{a} \in \mathbb{F}^{m}$ are zeros, then

$$
k_{m-1}(\mathbf{a})=(-1)^{m-v}(q-1)^{v-1} .
$$

If $v=0$, then we have the following well known bound by Deligne:

$$
\left|k_{m-1}(\mathbf{a})\right| \leq m q^{\frac{m-1}{2}} .
$$

Lemma 2.2. The dimension $k$ of $C(r, m)$ over $\mathbb{F}_{2}$ is $r m$ if $r m>4$. If $r=m=2$, then $k=2$.

Proof. Consider group homomorphism

$$
\Psi:\left(\mathbb{F}^{m},+\right) \longrightarrow C(r, m), \mathbf{a} \mapsto\left(\operatorname{tr}\left(f_{\mathbf{a}}\left(\mathbf{x}_{1}\right)\right), \ldots, \operatorname{tr}\left(f_{\mathbf{a}}\left(\mathbf{x}_{n}\right)\right)\right)
$$


If a belongs to $\operatorname{Ker}(\Psi)$ then $k_{m-1}(\mathbf{a})=(q-1)^{m-1}$. If $r m>4$, this can happen if and only if $\mathbf{a}=\mathbf{0}$, by Deligne's bound and by Lemma 2.1, and therefore $\psi$ is an isomorphism.

If $r=m=2$ and $a, b \in \mathbb{F}_{4}^{*}$, then $k_{m-1}((a, b))=k_{m-1}(a b)$. If $a b=1$, then $k_{m-1}(a b)=3$ and otherwise $k_{m-1}(a b)=-1$. Hence, in this case, $|\operatorname{Ker}(\Psi)|=4$ and consequently $|C(r, m)|=16 / 4=4$.

Remark. A different proof for this result is given in [4, Theorem 3.1]

The Hamming weight $w(c(\mathbf{a}))$ of codeword $c(\mathbf{a})$ is given by

$$
w(c(\mathbf{a}))=\sum_{\mathbf{x} \in\left(\mathbb{F}^{*}\right)^{m-1}} \frac{1}{2}\left(1-(-1)^{\mathrm{t} r\left(f_{\mathbf{a}}(\mathbf{x})\right)}\right)=\frac{1}{2}\left((q-1)^{m-1}-k_{m-1}(\mathbf{a})\right) .
$$

Next we express $w(c(\mathbf{a}))$ by means of a monomial exponential sum over $\mathbb{F}_{q^{m}}$. Let $e$ denote the canonical additive character of $\mathbb{F}_{q^{m}}$. Let $t=\left(q^{m}-1\right) /(q-1)$ and let $\mathrm{N}(\alpha):=\alpha^{t}$ denote the norm of $\alpha$ from $\mathbb{F}_{q^{m}}$ to $\mathbb{F}_{q}$. Let $\gamma$ be a primitive element of $\mathbb{F}_{q^{m}}$, and let

$$
s(\alpha)=\sum_{i=0}^{t-1} e\left(\alpha \gamma^{(q-1) i}\right)
$$

We have the following result from [9, Theorem 3]:

TheOREM 2.3. Let $\alpha \in \mathbb{F}_{q^{m}}^{*}$ and let $a=\mathrm{N}(\alpha)$. Then

$$
\sum_{x \in \mathbb{F}_{q^{m}}^{*}} e\left(\alpha x^{q-1}\right)=(-1)^{m-1}(q-1) k_{m-1}(a),
$$

or, equivalently,

$$
k_{m-1}(a)=(-1)^{m-1} s(\alpha) .
$$

LemMa 2.4. Let $\mathbf{a} \in\left(\mathbb{F}^{*}\right)^{m}$ and let $b=a_{1} \cdots a_{m}$. Let $g:=\mathrm{N}(\gamma)$ be a primitive element of $\mathbb{F}, i=\operatorname{ind}_{g}(b)$, and $\beta=\gamma^{i}$. Then

$$
\begin{aligned}
w(c(\mathbf{a})) & =\frac{1}{2}\left((q-1)^{m-1}-k_{m-1}(b)\right) \\
& =\frac{1}{2}\left((q-1)^{m-1}+(-1)^{m} s(\beta)\right) .
\end{aligned}
$$

Proof. The first equality follows easily by equation (2.2), and the second one then by Theorem 2.3 .

Let $S$ denote the range of $s\left(\gamma^{i}\right)$ as $i$ varies over the set $I:=\{0, \ldots, q-2\}$, and, for $j \in S$, let $N_{j}$ denote the number of elements $i$ in $I$ such that $s\left(\gamma^{i}\right)=j$, i.e.

$$
S=\left\{s\left(\gamma^{i}\right) \mid i \in I\right\}
$$

and

$$
N_{j}=\left|\left\{i \in I \mid s\left(\gamma^{i}\right)=j\right\}\right|
$$


Theorem 2.5. Assume $\mathrm{rm}>4$. For $\mathbf{a} \in \mathbb{F}^{m}$ let $v$ be the number of zero components of $\mathbf{a}$. If $v>0$, there are

$$
\left(\begin{array}{c}
m \\
v
\end{array}\right)(q-1)^{m-v} \text { codewords } c(\mathbf{a}) \text { of weight }\left((q-1)^{m-1}-(-1)^{m-v}(q-1)^{v-1}\right) / 2
$$

and otherwise, for each $j \in S$, there are

$$
N_{j}(q-1)^{m-1} \text { codewords } c(\mathbf{a}) \text { of weight }\left((q-1)^{m-1}+(-1)^{m} j\right) / 2
$$

in $C(r, m)$. Moreover, these are the only weights in $C(r, m)$.

Proof. First, for each $\mathbf{a} \in \mathbb{F}^{m}$, there exists exactly one codeword $c(\mathbf{a}) \in C(r, m)$, by isomorphism (2.1). If $v>0$ the claim follows now by Lemmas 2.1 and 2.4.

Assume $v=0$. For each $b \in \mathbb{F}^{*}$ there are exactly $(q-1)^{m-1}$ vectors a $\in\left(\mathbb{F}^{*}\right)^{m}$ such that the product of the components of a equals $b$. The second claim follows now by Lemma 2.4 since there is exactly one $i$ in $I$ such that $\mathrm{N}\left(\gamma^{i}\right)=b$. The last claim is now obvious.

COROLlaRY 2.6. The weights in $C(r, m)$ are divisible by $2^{\ell-1}$, where $\ell=\min \{r, m\}$.

Proof. Let $\alpha \in \mathbb{F}_{q^{m}}$. By [12, Theorem 2], the exponential sum

$$
\sum_{x \in \mathbb{F}_{q^{m}}} e\left(\alpha x^{q-1}\right)
$$

is divisible by $2^{\lceil r m / s\rceil}$ where $s$ is the binary weight of $q-1$. Now $s=r$, and therefore $\sum_{x \in \mathbb{F}_{q} m} e\left(\alpha x^{q-1}\right)=2^{m} z$ for some $z \in \mathbb{Z}$.

Let $\mathbf{a} \in\left(\mathbb{F}^{*}\right)^{m}$, and let $\beta \in \mathbb{F}_{q^{m}}^{*}$ such that $\mathrm{N}(\beta)=a_{1} \cdots a_{m}$. Now

$$
\begin{aligned}
(q-1) w(c(\mathbf{a})) & =\frac{1}{2}\left((q-1)^{m}+(-1)^{m}(q-1) s(\beta)\right) \\
& =\frac{1}{2}\left((q-1)^{m}+(-1)^{m} \sum_{x \in \mathbb{F}_{q^{m}}^{*}} e\left(\alpha x^{q-1}\right)\right) \\
& =\frac{1}{2}\left((q-1)^{m}+(-1)^{m} \sum_{x \in \mathbb{F}_{q^{m}}} e\left(\alpha x^{q-1}\right)-(-1)^{m}\right) \\
& =\frac{1}{2}\left(q^{m}-m q^{m-1}+\cdots+(-1)^{m-1} m q+(-1)^{m} 2^{m} z\right)
\end{aligned}
$$

and, as $q=2^{r}$, the claim follows in this case. If some of the components of $\mathbf{a}$ is zero, then it is easily seen that $2^{r-1}$ is a factor of $w(c(\mathbf{a}))$.

Remark. A different proof for this result is given in [3, Corollary 4.3].

To obtain the weight distribution of $C^{\perp}(r, m)$ we use the Pless power moment identity proved in [13] (see also e.g. [6, p. 131]):

TheOrem 2.7 (Power moment identity). Let $B$ be a binary linear $[n, k]$ code, and let $B_{i}$ (resp. $B_{i}^{\perp}$ ) denote the number of codewords of weight $i$ in $B$ (resp. in $\left.B^{\perp}\right)$. Then, for $h=0,1, \ldots$, we have:

$$
\sum_{i=0}^{n} i^{h} B_{i}=\sum_{i=0}^{n}(-1)^{i} B_{i}^{\perp} \sum_{t=0}^{h} t ! S(h, t) 2^{k-t}\left(\begin{array}{c}
n-i \\
n-t
\end{array}\right)
$$


where

$S(h, t):=\frac{1}{t !} \sum_{j=0}^{t}(-1)^{t-j}\left(\begin{array}{l}t \\ j\end{array}\right) j^{h} \quad$ (a Stirling number of the second kind),

and the binomial coefficient $\left(\begin{array}{l}u \\ v\end{array}\right)$ is defined to be zero whenever $v>u$ or $v<0$.

For a non-negative integer $j$ we denote by $M_{j}$ the $j$ th moment of the period $s\left(\gamma^{l}\right)$, or

$$
M_{j}:=\sum_{l=0}^{q-2} s\left(\gamma^{l}\right)^{j}
$$

Theorem 2.8. Assume $r m>4$, and let $w=1-q$. The number $C_{h}^{\perp}$ of codewords of weight $h$ in $C^{\perp}(r, m)$ is given by

$$
\begin{aligned}
q^{m} h ! C_{h}^{\perp}=f & \left(C_{0}^{\perp}, \ldots, C_{h-1}^{\perp}\right)+g\left(M_{0}, \ldots, M_{h}\right) \\
& +(-1)^{(m+1)(h+1)} w^{-h}\left(w^{m}\left(1-w^{m}\right)^{h}-\sum_{j=0}^{h}\left(\begin{array}{c}
h \\
j
\end{array}\right)(-1)^{j}\left(w^{j+1}-w^{h}\right)^{m}\right),
\end{aligned}
$$

where

$$
\begin{aligned}
& f\left(C_{0}^{\perp}, \ldots, C_{h-1}^{\perp}\right)=q^{m} \sum_{i=0}^{h-1}(-1)^{h+i+1} C_{i}^{\perp} \sum_{t=i}^{h} t ! S(h, t) 2^{h-t}\left(\begin{array}{c}
n-i \\
n-t
\end{array}\right) \\
& g\left(M_{0}, \ldots, M_{h}\right)=\sum_{j=0}^{h}\left(\begin{array}{l}
h \\
j
\end{array}\right)(-1)^{m j+h}(q-1)^{(m-1)(h-j+1)} M_{j} .
\end{aligned}
$$

Moreover, if $m=3$, the formula simplifies to

$$
\begin{aligned}
q^{3} h ! C_{h}^{\perp}=f & \left(C_{0}^{\perp}, \ldots, C_{h-1}^{\perp}\right)+g\left(M_{0}, \ldots, M_{h}\right) \\
& +3(q-1)^{2}(-q)^{h}\left((q-2)^{h}+(q-1)^{h-1}\right) .
\end{aligned}
$$

Proof. We choose $B=C(r, m)$ in the power moment identity. Then, by Theorem 2.5,

$$
\begin{aligned}
\sum_{i=0}^{n} i^{h} C_{i} & =\sum_{v=1}^{m}\left(\begin{array}{c}
m \\
v
\end{array}\right)(q-1)^{m-v} 2^{-h}\left((q-1)^{m-1}-(-1)^{m-v}(q-1)^{v-1}\right)^{h} \\
& +\sum_{l=0}^{q-2} 2^{-h}(q-1)^{m-1}\left((q-1)^{m-1}+(-1)^{m} s\left(\gamma^{l}\right)\right)^{h}=: S_{1}+S_{2}
\end{aligned}
$$

where

$$
2^{h} S_{1}=\sum_{v=1}^{m}\left(\begin{array}{c}
m \\
v
\end{array}\right)(q-1)^{m-v}\left((q-1)^{m-1}-(-1)^{m-v}(q-1)^{v-1}\right)^{h}
$$

and

$$
2^{h} S_{2}=(q-1)^{m-1} \sum_{l=0}^{q-2}\left((q-1)^{m-1}+(-1)^{m} s\left(\gamma^{l}\right)\right)^{h}
$$


First, we manipulate $S_{2}$ somewhat:

$$
\begin{aligned}
2^{h} S_{2} & =(q-1)^{m-1} \sum_{l=0}^{q-2} \sum_{j=0}^{h}\left(\begin{array}{l}
h \\
j
\end{array}\right)(-1)^{m j} s\left(\gamma^{l}\right)^{j}(q-1)^{(m-1)(h-j)} \\
& =\sum_{j=0}^{h}\left(\begin{array}{l}
h \\
j
\end{array}\right)(-1)^{m j}(q-1)^{(m-1)(h-j+1)} \sum_{l=0}^{q-2} s\left(\gamma^{l}\right)^{j} \\
& =\sum_{j=0}^{h}\left(\begin{array}{l}
h \\
j
\end{array}\right)(-1)^{m j}(q-1)^{(m-1)(h-j+1)} M_{j} .
\end{aligned}
$$

Secondly we consider $S_{1}$. If $m=3$, then

$$
2^{h} S_{1}=3(q-1)^{2} q^{h}\left((q-2)^{h}+(q-1)^{h-1}\right) .
$$

Next we write $S_{1}$ in the form from which we can derive explicit formulae for the number of low-weight codewords in the duals $C^{\perp}(r, m)$ for an arbitrary integer $m \geq 2$ :

$$
\begin{aligned}
2^{h} S_{1} & =(q-1)^{m-1} \sum_{v=1}^{m}\left(\begin{array}{c}
m \\
v
\end{array}\right)(q-1)^{(v-1)(h-1)}\left((q-1)^{m-v}+(-1)^{m-v-1}\right)^{h} \\
& =(q-1)^{m-1} \sum_{v=1}^{m}\left(\begin{array}{c}
m \\
v
\end{array}\right)(q-1)^{(v-1)(h-1)}(-1)^{(m-v-1) h}\left(1-(1-q)^{m-v}\right)^{h} \\
& =(-1)^{(m-1) h}(-w)^{m-1} \sum_{j=0}^{h}\left(\begin{array}{c}
h \\
j
\end{array}\right)(-1)^{j} \sum_{v=1}^{m}\left(\begin{array}{c}
m \\
v
\end{array}\right)(-1)^{v h}(-w)^{(v-1)(h-1)} w^{(m-v) j} \\
& =(-1)^{(m-1) h}(-w)^{-h} \sum_{j=0}^{h}\left(\begin{array}{c}
h \\
j
\end{array}\right)(-1)^{j} \sum_{v=1}^{m}\left(\begin{array}{c}
m \\
v
\end{array}\right) w^{v h}(-w)^{m-v} w^{(m-v) j} \\
& =(-1)^{(m-1) h+m}(-w)^{-h} \sum_{j=0}^{h}\left(\begin{array}{c}
h \\
j
\end{array}\right)(-1)^{j} \sum_{v=1}^{m}\left(\begin{array}{c}
m \\
v
\end{array}\right)(-1)^{v} w^{v h} w^{(m-v)(j+1)} \\
& =(-1)^{m(h+1)} w^{-h} \sum_{j=0}^{h}\left(\begin{array}{c}
h \\
j
\end{array}\right)(-1)^{j}\left(\left(w^{j+1}-w^{h}\right)^{m}-w^{m(j+1)}\right) .
\end{aligned}
$$

Since

$$
\begin{aligned}
\sum_{j=0}^{h}\left(\begin{array}{l}
h \\
j
\end{array}\right)(-1)^{j} w^{m(j+1)} & =(1-q)^{m} \sum_{j=0}^{h}\left(\begin{array}{l}
h \\
j
\end{array}\right)(-1)^{j}(1-q)^{m j} \\
& =w^{m}\left(1-w^{m}\right)^{h}
\end{aligned}
$$

we have

$$
\begin{aligned}
2^{h} S_{1}= & (-1)^{m(h+1)} w^{-h} \sum_{j=0}^{h}\left(\begin{array}{l}
h \\
j
\end{array}\right)(-1)^{j}\left(w^{j+1}-w^{h}\right)^{m} \\
& -(-1)^{m(h+1)} w^{m-h}\left(1-w^{m}\right)^{h} .
\end{aligned}
$$


As the left hand side of the power moment identity equals $S_{1}+S_{2}$, and the right hand side equals

$$
\begin{aligned}
& \sum_{i=0}^{n}(-1)^{i} C_{i}^{\perp} \sum_{t=0}^{h} t ! S(h, t) 2^{r m-t}\left(\begin{array}{c}
n-i \\
n-t
\end{array}\right) \\
& =\sum_{i=0}^{h}(-1)^{i} C_{i}^{\perp} \sum_{t=i}^{h} t ! S(h, t) 2^{r m-t}\left(\begin{array}{c}
n-i \\
n-t
\end{array}\right) \\
& =\frac{q^{m}}{2^{h}} \sum_{i=0}^{h}(-1)^{i} C_{i}^{\perp} \sum_{t=i}^{h} t ! S(h, t) 2^{h-t}\left(\begin{array}{c}
n-i \\
n-t
\end{array}\right),
\end{aligned}
$$

the claims follow now easily.

3. The minimum distance of $C^{\perp}(r, m)$. To determine the minimum distance of $C^{\perp}(r, m)$ we need some auxiliary results. We recall that $t=\left(q^{m}-1\right) /(q-1)$ and $\mathbb{F}_{q^{m}}^{*}=\langle\gamma\rangle$.

Lemma 3.1. The first four moments $M_{j}$ in Theorem 2.8 are given by

$$
\begin{aligned}
& M_{0}=q-1, M_{1}=-1, M_{2}=q^{m}-t, \\
& M_{3}=\frac{\left|\mathcal{X}\left(\mathbb{F}_{q^{m}}\right)\right|-3(q-1)}{(q-1)^{2}} q^{m}-t^{2},
\end{aligned}
$$

where $\left|\mathcal{X}\left(\mathbb{F}_{q^{m}}\right)\right|$ is the number of rational points on the projective curve $\mathcal{X}$ over $\mathbb{F}_{q^{m}}$ defined by the equation

$$
\mathcal{X}: x^{q-1}+y^{q-1}+z^{q-1}=0 .
$$

Proof. Obviously $M_{0}=q-1$, and

$$
M_{1}=\sum_{l=0}^{q-2} s\left(\gamma^{l}\right)=\frac{q-1}{q^{m}-1} \sum_{l=0}^{q^{m}-2} s\left(\gamma^{l}\right)=\frac{1}{t} \sum_{i=0}^{t-1} \sum_{l=0}^{q^{m}-2} e\left(\gamma^{(q-1) i} \gamma^{l}\right)=-\frac{t}{t},
$$

where the last equality follows by the orthogonality of characters. To prove the formula for $M_{2}$ we count the number $N$ of solutions of the equation $x+y=0$ in the group $H$ of $(q-1)$ th powers in $\mathbb{F}_{q^{m}}^{*}$. On the one hand $N=t$, and on the other hand, by the orthogonality of characters

$$
\begin{aligned}
q^{m} t & =\sum_{x, y \in H} \sum_{u \in \mathbb{F}_{q}^{m}} e(u(x+y))=t^{2}+\sum_{u \in \mathbb{F}_{q^{m}}^{*}}\left(\sum_{x \in H} e(u x)\right)^{2}=t^{2}+t \sum_{l=0}^{q-2} s\left(\gamma^{l}\right)^{2} \\
& =t^{2}+t M_{2}
\end{aligned}
$$

from which the formula for $M_{2}$ follows.

Let $N$ denote the number of solutions of equation

$$
x^{q-1}+y^{q-1}+z^{q-1}=0
$$

in $\mathbb{F}_{q^{m}}^{3}$. It is easy to see $\left(\left[9\right.\right.$, Section 3]) that $N=N_{m}^{\prime}+N_{m}$, where

$$
N_{m}^{\prime}=3(q-1)\left(q^{m}-1\right)+1
$$


and

$$
\begin{aligned}
q^{m} N_{m} & =\sum_{u \in \mathbb{F}_{q}^{*}}\left(\sum_{x \in \mathbb{F}_{q^{m}}^{*}} e\left(u x^{q-1}\right)\right)^{3}+\left(q^{m}-1\right)^{3} \\
& =(q-1)^{3} \sum_{u \in \mathbb{F}_{q^{m}}^{*}} s(u)^{3}+\left(q^{m}-1\right)^{3} \\
& =(q-1)^{3} t M_{3}+\left(q^{m}-1\right)^{3},
\end{aligned}
$$

and consequently,

$$
q^{m} N=3(q-1)\left(q^{m}-1\right) q^{m}+q^{m}+(q-1)^{3} t M_{3}+\left(q^{m}-1\right)^{3} .
$$

Since $\left|\mathcal{X}\left(\mathbb{F}_{q^{m}}\right)\right|=(N-1) /\left(q^{m}-1\right)$, we obtain

$$
(q-1)^{3} \frac{q^{m}-1}{q-1} M_{3}=q^{m}\left(q^{m}-1\right)\left|\mathcal{X}\left(\mathbb{F}_{q^{m}}\right)\right|-3(q-1)\left(q^{m}-1\right) q^{m}-\left(q^{m}-1\right)^{3},
$$

which simplifies to

$$
(q-1)^{2} M_{3}=q^{m}\left|\mathcal{X}\left(\mathbb{F}_{q^{m}}\right)\right|-3(q-1) q^{m}-\left(q^{m}-1\right)^{2} .
$$

Since $\left(q^{m}-1\right)^{2}=(q-1)^{2} t^{2}$, we see that the claim is true also for $M_{3}$.

THEOREM 3.2. The minimum distance of $C^{\perp}(r, m)$ is at least three. Moreover, if $r m>4$, the number $C_{3}^{\perp}$ of weight three codewords in $C^{\perp}(r, m)$ is given by

$$
C_{3}^{\perp}=\frac{(q-1)^{m-3}\left((q-2)^{m}+(-1)^{m}\left(q^{m}+3 q-\left|\mathcal{X}\left(\mathbb{F}_{q^{m}}\right)\right|-5\right)\right)}{6}
$$

Proof. Let $n=(q-1)^{m-1}$, and let $\mathbf{c} \in C^{\perp}(r, m)$. If $w(\mathbf{c})=2$ then

$$
\operatorname{tr}\left(f_{\mathbf{a}}\left(\mathbf{x}_{i}\right)+f_{\mathbf{a}}\left(\mathbf{x}_{j}\right)\right)=\operatorname{tr}\left(f_{\mathbf{a}}\left(\mathbf{x}_{i}\right)\right)+\operatorname{tr}\left(f_{\mathbf{a}}\left(\mathbf{x}_{j}\right)\right)=0
$$

for some $1 \leq i<j \leq n$, say $i=1, j=2$, and for all $\mathbf{a} \in \mathbb{F}_{q}^{m}$. Let $1 \leq l \leq m-1$ be the index of the coordinate place where $\mathbf{x}_{1}$ and $\mathbf{x}_{2}$ differ, say $l=1$. By choosing $\mathbf{a}=(a, 0, \ldots, 0)$ we have $\operatorname{tr}(a(x+y))=0$ for all $a \in \mathbb{F}_{q}$, and for some $x, y \in \mathbb{F}_{q}^{*}$ with $x \neq y$. (Here $x$ and $y$ are the first components of $\mathbf{x}_{1}$ and $\mathbf{x}_{2}$.) This contradicts the surjectivity of $\mathrm{t} r$. A similar argument also proves that $w(\mathbf{c}) \neq 1$.

Next we use Theorem 2.8 and Lemma 3.1 to prove the claimed formula for $C_{3}^{\perp}$. First,

$$
\begin{aligned}
f\left(C_{0}^{\perp}, C_{1}^{\perp}, C_{2}^{\perp}\right) & =f(1,0,0)=q^{m} \sum_{t=0}^{3} t ! S(3, t) 2^{3-t}\left(\begin{array}{c}
n \\
n-t
\end{array}\right) \\
& =q^{m}(4 n+6 n(n-1)+(n-2)(n-1) n) \\
& =(q-1)^{2 m-2}\left((q-1)^{m-1}+3\right) q^{m},
\end{aligned}
$$

and second,

$$
\begin{aligned}
& g\left(M_{0}, M_{1}, M_{2}, M_{3}\right)=\sum_{j=0}^{3}\left(\begin{array}{l}
3 \\
j
\end{array}\right)(-1)^{m j+h}(q-1)^{(m-1)(h-j+1)} M_{j} \\
& =-(q-1)^{4 m-3}+3(-1)^{m}(q-1)^{3(m-1)}-3(q-1)^{2(m-1)}\left(q^{m}-\frac{q^{m}-1}{q-1}\right) \\
& \quad-(-1)^{m}(q-1)^{m-1}\left(\frac{\left|\mathcal{X}\left(\mathbb{F}_{q^{m}}\right)\right|-3(q-1)}{(q-1)^{2}} q^{m}-\frac{\left(q^{m}-1\right)^{2}}{(q-1)^{2}}\right),
\end{aligned}
$$


or, equivalently,

$$
\begin{aligned}
& (q-1)^{3-m} g\left(M_{0}, M_{1}, M_{2}, M_{3}\right)=(-1)^{m}-3(q-1)^{m}+3(-1)^{m}(q-1)^{2 m} \\
& -(q-1)^{3 m}+\left((-1)^{m}\left(q^{m}+3 q-\left|\mathcal{X}\left(\mathbb{F}_{q^{m}}\right)\right|-5\right)-3(q-2)(q-1)^{m}\right) q^{m} .
\end{aligned}
$$

Finally, since

$$
\begin{gathered}
(q-1)^{3-m} w^{-3}\left(w^{m}\left(1-w^{m}\right)^{3}-\sum_{j=0}^{3}\left(\begin{array}{l}
3 \\
j
\end{array}\right)\left(w^{j+1}-w^{3}\right)^{m}\right)= \\
-(-1)^{m}+3(q-1)^{m}-3(-1)^{m}(q-1)^{2 m}+(q-1)^{3 m} \\
-\left((q-1)^{m}+3\right)(q-1)^{m} q^{m}+(q-2)^{m} q^{m},
\end{gathered}
$$

we obtain

$$
6 C_{3}^{\perp}=(q-1)^{m-3}\left((q-2)^{m}+(-1)^{m}\left(q^{m}+3 q-\left|\mathcal{X}\left(\mathbb{F}_{q^{m}}\right)\right|-5\right)\right),
$$

by Theorem 2.8.

Example 3.3. If $r=m=2$ then $n=3$, and therefore the minimum distance of $C^{\perp}(2,2)$ is three. Hence, $C^{\perp}(2,2)$ is a repetition code.

Example 3.4. Consider the Melas code $C^{\perp}(r, 2)$. By [9, Theorem 1]

$$
\left|\mathcal{X}\left(\mathbb{F}_{q^{2}}\right)\right|=\left(1-(-1)^{r}\right)(q-1)^{2}+3(q-1),
$$

and consequently

$$
C_{3}^{\perp}=\left(1+(-1)^{r}\right)(q-1) / 6,
$$

which is in accordance with [14, Table 6.1].

Example 3.5. Consider code $C^{\perp}(r, 3)$. By [9, Theorem 2]

$$
\left|\mathcal{X}\left(\mathbb{F}_{q^{3}}\right)\right|=\left(2 q+1-(-1)^{r}\right)(q-1)^{2}+3(q-1),
$$

and therefore

$$
C_{3}^{\perp}=\left(2 q-5-(-1)^{r}\right)(q-1)^{2} / 6 .
$$

Remark. By generalizing the argument used in the proof of Theorem 3.2 to prove the non-existence of weight two codewords, it is easy to see that a check matrix for $C^{\perp}(r, m)$ is $\left(\mathbf{y}_{1}^{T} \mathbf{y}_{2}^{T} \ldots \mathbf{y}_{n}^{T}\right)$ where $\mathbf{y}_{i}=\left(\mathbf{x}_{i} z_{i}\right)$ and $z_{i}$ is the product of the inverses of the components of $\mathbf{x}_{i}$.

We shall see soon that the minimum distance of $C^{\perp}(r, m)$ is always three if $m>2$. It will turn out that Theorem 3.2 together with the Hasse-Weil bound prove most of the cases. On the other hand, in case $m=4$ it is too weak, and we shall use the following upper bound:

LEMMA 3.6.

$$
\left|\mathcal{X}\left(\mathbb{F}_{q^{m}}\right)\right|<q^{m}+3 q+(q-1)^{3} m^{3} q^{\frac{m-3}{2}}-4
$$


Proof. As we pointed out in the proof of Lemma 3.1, the number of solutions $N$ of (3.1) satisfies

$$
q^{m} N=(q-1)^{3}\left(\sum_{u \in \mathbb{F}_{q^{m}}^{*}} e\left(u x^{q-1}\right)\right)+\left(q^{m}-1\right)^{3}+3(q-1)\left(q^{m}-1\right) q^{m}+q^{m},
$$

and then it is easily seen (see $[9$, Section 3]) that

$$
q^{m} N=(-1)^{m-1} t(q-1)^{3} \sum_{u \in \mathbb{F}_{q}^{*}} k_{m-1}(u)^{3}+\left(q^{m}-1\right)^{3}+3(q-1)\left(q^{m}-1\right) q^{m}+q^{m} .
$$

Since $\left|\mathcal{X}\left(\mathbb{F}_{q^{m}}\right)\right|=(N-1) /\left(q^{m}-1\right)$ we obtain

$$
q^{m}\left|\mathcal{X}\left(\mathbb{F}_{q^{m}}\right)\right|=(-1)^{m-1}(q-1)^{2} \sum_{u \in \mathbb{F}_{q}^{*}} k_{m-1}(u)^{3}+\left(q^{m}-1\right)^{2}+3(q-1) q^{m} .
$$

Now Deligne's bound gives the inequality

$$
\left|\sum_{u \in \mathbb{F}_{q}^{*}} k_{m-1}(u)^{3}\right| \leq(q-1) m^{3} q^{\frac{3(m-1)}{2}},
$$

and therefore

$$
\left|\mathcal{X}\left(\mathbb{F}_{q^{m}}\right)\right| \leq(q-1)^{3} m^{3} q^{\frac{m-3}{2}}+q^{m}-2+3(q-1)+q^{-m} .
$$

口

TheOREM 3.7. The minimum distance of $C^{\perp}(r, m)$ is three unless $r$ is odd and $m=2$, in which case it is at least five.

Proof. Assume $m=2$. If $r=2$ the minimum distance $d=3$ by Example 3.3, and if $r>2$, then it is well known that $d=3$ or $d \geq 5$ according as $r$ is even or odd (see e.g. $[14])$.

CLAiM. If $m>2$ then $d=3$.

If $m=3$ the Claim is true by Example 3.5. Assume $m>3$. To prove the Claim it is enough, by Theorem 3.2 , to show that

$$
\epsilon:=(q-2)^{m}+(-1)^{m}\left(q^{m}+3 q-\left|\mathcal{X}\left(\mathbb{F}_{q^{m}}\right)\right|-5\right)
$$

is positive. By separating the cases according to the parity of $m$, and by using the Hasse-Weil bounds

$$
q^{m}+1-(q-2)(q-3) q^{\frac{m}{2}} \leq\left|\mathcal{X}\left(\mathbb{F}_{q^{m}}\right)\right| \leq q^{m}+1+(q-2)(q-3) q^{\frac{m}{2}},
$$

we obtain

$$
\epsilon>(q-2)^{m}-(q-2)(q-3) q^{\frac{m}{2}}-3 q-6,
$$

which is obviously positive if $m \geq 5$ and $r \geq 3$ (i.e. $q \geq 8$ ).

Assume $m=4$ and $\epsilon=0$. Then, by Lemma 3.6, we must have

$$
\begin{gathered}
(q-2)^{4}<64 \sqrt{q}(q-1)^{3}+1 \Leftrightarrow \\
(q-1)^{4}<64 \sqrt{q}(q-1)^{3}+4(q-1)^{3}-6(q-1)^{2}+4(q-1) \Leftrightarrow \\
q-1<64 \sqrt{q}+4-\frac{6}{q-1}+\frac{4}{(q-1)^{2}}<64 \sqrt{q}+5 . \\
10
\end{gathered}
$$




\begin{tabular}{c|c}
$r$ & $\epsilon$ \\
\hline 3 & $2^{3} \cdot 3 \cdot 7^{2}$ \\
4 & $2 \cdot 3^{3} \cdot 5^{2} \cdot 37$ \\
5 & $2^{4} \cdot 3^{2} \cdot 5 \cdot 31^{2}$ \\
6 & $2 \cdot 3^{5} \cdot 7^{2} \cdot 641$ \\
7 & $2^{3} \cdot 3^{2} \cdot 7 \cdot 31 \cdot 127^{2}$ \\
8 & $2 \cdot 3^{3} \cdot 5^{2} \cdot 7 \cdot 17^{2} \cdot 1531$ \\
9 & $2^{8} \cdot 3 \cdot 5 \cdot 7^{2} \cdot 67 \cdot 73^{2}$ \\
10 & $2 \cdot 3^{3} \cdot 11^{5} \cdot 31^{2} \cdot 131$ \\
11 & $2^{3} \cdot 3^{3} \cdot 11 \cdot 23^{2} \cdot 89^{2} \cdot 1759$ \\
12 & $2 \cdot 3^{5} \cdot 5^{2} \cdot 7^{2} \cdot 13^{2} \cdot 113 \cdot 24709$
\end{tabular}

The inequality $q-6<64 \sqrt{q}$ implies that we must have $q \leq 2^{12}$ i.e. $r \leq 12$. Hence, if $m=4$ and $r>12$ the minimum distance is three. In the cases $m=4,3 \leq r \leq 12$, we have verified this by calculating $\left|\mathcal{X}\left(\mathbb{F}_{q^{4}}\right)\right|$ numerically (see Table 3.1).

In the remaining cases $r=2, m \geq 4$, the Claim follows by Theorem 4.3 below, by which $C_{3}^{\perp}=3^{m-3}\left(2^{m-1} \pm 1\right)$.

We computed $\left|\mathcal{X}\left(\mathbb{F}_{q^{4}}\right)\right|$ by using (3.2). In the calculation of the three dimensional Kloosterman sums $k_{3}(a)$ over $\mathbb{F}_{q}, q=2^{r}$ with $3 \leq r \leq 12$, we took advantage of the following result by Carlitz from [1] which related two and one dimensional Kloosterman sums:

ThEOREM 3.8. For any $a \in \mathbb{F}_{q}^{*}$, we have

$$
k_{2}(a)=k(a)^{2}-q,
$$

where $k(a):=k_{1}(a)$.

By Theorem 3.8 we have

$$
\begin{aligned}
k_{3}(a) & =\sum_{x, y, z \in \mathbb{F}_{q}^{*}} \chi\left(x+y+z+a(x y z)^{-1}\right)=\sum_{x \in \mathbb{F}_{q}^{*}} \chi(x) k_{2}\left(a x^{-1}\right) \\
& =\sum_{x \in \mathbb{F}_{q}^{*}} \chi(x) k\left(a x^{-1}\right)^{2}-q \sum_{x \in \mathbb{F}_{q}^{*}} \chi(x) \\
& =\sum_{x \in \mathbb{F}_{q}^{*}} \chi(x) k\left(a x^{-1}\right)^{2}+q,
\end{aligned}
$$

and now it is easy to see that

$$
\begin{aligned}
k_{3}(a) & =2 \sum_{\substack{x \in \mathbb{F}_{q}^{*} \\
\operatorname{tr}(x)=0}} k\left(a x^{-1}\right)^{2}-\sum_{x \in \mathbb{F}_{q}^{*}} k\left(a x^{-1}\right)^{2}+q \\
& =2 \sum_{\substack{x \in \mathbb{F}_{*}^{*} \\
\operatorname{tr}(x)=0}} k\left(a x^{-1}\right)^{2}-\left(q^{2}-q-1\right)+q .
\end{aligned}
$$

By tabulating the traces of elements of $\mathbb{F}_{q}^{*}$, the indices of those elements of $\mathbb{F}_{q}^{*}$ having the trace equal to zero, and then, the range of $k(u)$ as $u$ varies over $\mathbb{F}_{q}^{*}$, before using the formula above, the data of Table 3.1 can quickly be verified.

Remark. The traces were calculated by making use of [4, Theorem 5.1]. 
4. The weight distribution of $C(2, m)$ and $C^{\perp}(2, m)$. In this section we assume that $m>2$. Let $\gamma$ be a primitive element of $\mathbb{F}_{2^{2 m}}$. To determine the weight distribution of $C(2, m)$ and $C^{\perp}(2, m)$ we need the following result which has been proved already in [2] (see e.g. [8] for a different proof).

Lemma 4.1. Let $\alpha \in \mathbb{F}_{2^{2 m}}^{*}$. Then

$$
\sum_{x \in \mathbb{F}_{22 m}^{*}} e\left(\alpha x^{3}\right)= \begin{cases}(-1)^{m} 2^{m}-1 & \text { if } 3 \nmid \text { ind } d_{\gamma} \alpha, \\ (-1)^{m+1} 2^{m+1}-1 & \text { if } 3 \mid \text { ind } d_{\gamma} \alpha .\end{cases}
$$

Lemma 4.1 together with Theorem 2.5 give the weight distribution of $C(2, m)$ :

ThEOREM 4.2. The weight distribution of $C(2, m)$ is given in the following table, where $v$ runs over the integers $1, \ldots, m$.

\begin{tabular}{c|c} 
weight & frequency \\
\hline$\frac{3^{m-1}-(-1)^{m-v} 3^{v-1}}{2}$ & $\left(\begin{array}{c}m \\
v\end{array}\right) 3^{m-v}$ \\
$\frac{1}{2}\left(3^{m-1}+\frac{2^{m}-(-1)^{m}}{3}\right)$ & $2 \cdot 3^{m-1}$ \\
$\frac{1}{2}\left(3^{m-1}-\frac{2^{m+1}+(-1)^{m}}{3}\right)$ & $3^{m-1}$
\end{tabular}

THEOREM 4.3. For every non-negative integer $h$ the number $C_{h}^{\perp}$ of codewords of weight $h$ in the dual $C^{\perp}(2, m)$ of $C(2, m)$ is given by the recursion of Theorem 2.8 with

$$
M_{j}=2\left(\frac{(-2)^{m}-1}{3}\right)^{j}+\left(\frac{(-2)^{m+1}-1}{3}\right)^{j} \quad \forall j=0,1, \ldots
$$

Especially,

$$
\begin{aligned}
& C_{0}^{\perp}=1, C_{1}^{\perp}=C_{2}^{\perp}=0, C_{3}^{\perp}=3^{m-3}\left(2^{m-1} \pm 1\right), \\
& C_{4}^{\perp}=3^{m-5}\left(\frac{7^{m}-3^{m+3}+66}{8}+3 \cdot 2^{2 m-2} \pm 2^{m}\right), \\
& C_{5}^{\perp}=3^{m-6}\left(\left(5^{m-1} \pm 6\right) 2^{2 m-3}-3^{m+2} 2^{m-2}+2^{3 m-2}+7 \cdot 2^{m+1} \pm \frac{55-3^{m+1}}{2}\right),
\end{aligned}
$$

where $\pm=(-1)^{m}$.

Proof. By Lemma 4.1 the moments $M_{j}$ in Theorem 2.8 are of the claimed form, the claimed formulae for the low-weight codewords can be verified e.g. by using Mathematica. $\mathrm{\square}$

Remark. In a similar manner as was done above, the weight distribution of the codes $C(r, m)$ and $C^{\perp}(r, m)$ with $r=3$ and $r=4$ can be calculated as well.

5. The weight distribution of $C(r, 3)$ and $C^{\perp}(r, 3)$. In this section we assume that $r>2$. Let $\gamma$ be a primitive element of $\mathbb{F}_{q^{3}}$, and let $g=\mathrm{N}(\gamma)$ be a primitive element of $\mathbb{F}=\mathbb{F}_{q}$.

Now, by Theorems 2.3 and 3.8 , we have the following:

LEMMA 5.1. For each integer $i$ satisfying $0 \leq i \leq q-2$, we have

$$
s\left(\gamma^{i}\right)=k\left(g^{i}\right)^{2}-q .
$$

Hence, the question about the distribution of the values of $s\left(\gamma^{i}\right)$ is equivalent to the question about the distribution of the values of (one dimensional) Kloosterman 
sums over $\mathbb{F}^{*}$. This question has been answered by Lachaud and Wolfmann in $[5$, Theorem 3.4 and Proposition 9.1]:

ThEOREM 5.2. The set of values $S$ of $k(a)$ as a runs over $\mathbb{F}_{q}^{*}$ is

$$
S=\{j \in \mathbb{Z}|| j \mid<2 \sqrt{q} \text { and } j \equiv-1 \quad(\bmod 4)\} .
$$

Moreover, each value $j \in S$ is attained exactly $H\left(j^{2}-4 q\right)$ times where $H(d)$ is the Kronecker class number of $d$.

As a corollary we obtain, by using Theorem 2.5, the weight distribution of $C(r, 3)$ :

TheOREM 5.3. The weight distribution of $C(r, 3)$ is given in the following table where $j$ runs over the set $\left\{|j|<2^{(r+2) / 2}\right.$ and $\left.j \equiv-1 \quad(\bmod 4)\right\}$ :

\begin{tabular}{c|c} 
weight & frequency \\
\hline 0 & 1 \\
$2^{r}\left(2^{r-1}-1\right)$ & $3\left(2^{r}-1\right)^{2}$ \\
$2^{r-1}\left(2^{r}-1\right)$ & $3\left(2^{r}-1\right)$ \\
$\left(2^{r}\left(2^{r}-1\right)-j^{2}+1\right) / 2$ & $H\left(j^{2}-2^{r+2}\right)\left(2^{r}-1\right)^{2}$
\end{tabular}

To give the weight distribution of $C^{\perp}(r, 3)$ we denote by $K_{h}$ the $h$ th moment of the Kloosterman sum $k(a)$ over the field $\mathbb{F}$, i.e.

$$
K_{h}=\sum_{a \in \mathbb{F}^{*}} k(a)^{h}
$$

and use the following result from [10] which was proved by using results from [14]:

Theorem 5.4. Let $q=2^{r}$. Then

$$
\begin{aligned}
& K_{0}=q-1, \quad K_{1}=1, \quad K_{2}=q^{2}-q-1, \quad K_{3}= \pm q^{2}+2 q+1, \\
& K_{4}=2 q^{3}-2 q^{2}-3 q-1, \\
& K_{5}=\left(t_{7} \pm 4\right) q^{3}+5 q^{2}+4 q+1, \\
& K_{6}=5 q^{4}-\left(5+(-1)^{r}\right) q^{3}-9 q^{2}-5 q-1, \\
& K_{7}=\left(t_{9}+6 t_{7} \pm 14+1\right) q^{4}+14 q^{3}+14 q^{2}+6 q+1, \\
& K_{8}=14 q^{5}-(15 \pm 7) q^{4}-28 q^{3}-20 q^{2}-7 q-1, \\
& K_{9}=\left(t_{11}+8 t_{9}+27 t_{7}+8 \pm 48\right) q^{5}+42 q^{4}+48 q^{3}+27 q^{2}+8 q+1, \\
& K_{10}=42 q^{6}-(51 \pm 35) q^{5}-90 q^{4}-75 q^{3}-35 q^{2}-9 q-1+2048 \tau(q / 4)-\tau(q),
\end{aligned}
$$

where \pm denotes $(-1)^{r}, t_{7}=\alpha_{7}^{r}+\bar{\alpha}_{7}^{r}$ with $\alpha_{7}=(1+\sqrt{-15}) / 4, t_{9}=\alpha_{9}^{r}+\bar{\alpha}_{9}^{r}$ with $\alpha_{9}=(-5+\sqrt{-39}) / 8, t_{11}=\beta_{11}^{r}+\bar{\beta}_{11}^{r}+\eta_{11}^{r}+\bar{\eta}_{11}^{r}$, with $\beta_{11}=(-3+\sqrt{505}+$ $\sqrt{-510-6 \sqrt{505}}) / 32, \eta_{11}=(-3-\sqrt{505}+\sqrt{-510+6 \sqrt{505}}) / 32$, and $\tau$ is the Ramanujan's tau-function.

Remark. It is not hard to see that

$$
\tau(q)-2048 \tau(q / 4)=\mu_{2}^{r}+\bar{\mu}_{2}^{r}=D_{r}(-24,2048),
$$

where $\mu_{2}=-12+4 \sqrt{-119}$ and $D_{r}(x, 2048)$ is the Dickson polynomial of the first kind of degree $r$ with parameter 2048 (see [11, Section 2]).

THEOREM 5.5. For every non-negative integer $h$ the number $C_{h}^{\perp}$ of codewords of weight $h$ in the dual $C^{\perp}(r, 3)$ of $C(r, 3)$ is given by

$$
\begin{gathered}
q^{3} h ! C_{h}^{\perp}=f\left(C_{0}^{\perp}, \ldots, C_{h-1}^{\perp}\right)+g\left(M_{0}, \ldots, M_{h}\right)+ \\
3(q-1)^{2}(-q)^{h}\left((q-2)^{h}+(q-1)^{h-1}\right),
\end{gathered}
$$


where

$$
\begin{gathered}
f\left(C_{0}^{\perp}, \ldots, C_{h-1}^{\perp}\right)=q^{3} \sum_{i=0}^{h-1}(-1)^{h+i+1} C_{i}^{\perp} \sum_{t=i}^{h} t ! S(h, t) 2^{h-t}\left(\begin{array}{c}
n-i \\
n-t
\end{array}\right), \\
g\left(M_{0}, \ldots, M_{h}\right)=\sum_{j=0}^{h}\left(\begin{array}{c}
h \\
j
\end{array}\right)(-1)^{j+h}(q-1)^{2(h-j+1)} \sum_{i=0}^{j}\left(\begin{array}{c}
j \\
i
\end{array}\right)(-q)^{j-i} K_{2 i} .
\end{gathered}
$$

Especially,

$$
\begin{aligned}
& C_{0}^{\perp}=1, C_{1}^{\perp}=C_{2}^{\perp}=0, C_{3}^{\perp}=(q-1)^{2}(2 q-5 \mp 1) / 3 !, \\
& C_{4}^{\perp}=(q-1)^{2}\left(q^{3}-6 q^{2}+(17 \mp 3) q-24\right) / 4 !, \\
& C_{5}^{\perp}=(q-1)^{2}\left(q^{5}-8 q^{4}+14 q^{3}+24 q^{2}-4(7 \pm 5) q-109 \mp 10\right. \\
& \left.+(2048 \tau(q / 4)-\tau(q)) / q^{3}\right) / 5 ! .
\end{aligned}
$$

Proof. The moments $M_{j}$ in Theorem 2.8 are, by Lemma 5.1, of the form

$$
\begin{aligned}
M_{j} & =\sum_{l=0}^{q-2}\left(k\left(g^{l}\right)^{2}-q\right)^{j}=\sum_{l=0}^{q-2} \sum_{i=0}^{j}\left(\begin{array}{l}
j \\
i
\end{array}\right) k\left(g^{l}\right)^{2 i}(-q)^{j-i} \\
& =\sum_{i=0}^{j}\left(\begin{array}{l}
j \\
i
\end{array}\right)(-q)^{j-i} \sum_{l=0}^{q-2} k\left(g^{l}\right)^{2 i} \\
& =\sum_{i=0}^{j}\left(\begin{array}{l}
j \\
i
\end{array}\right)(-q)^{j-i} K_{2 i},
\end{aligned}
$$

and the first claim follows now by Theorem 2.8. The validity of the formulae for the number of low-weight codewords can be verified by using Mathematica.

Remark. By Theorem 5.2, moments $K_{h}$ can be calculated effectively for each non-negative integer $h$ by

$$
K_{h}=\sum_{\substack{|j|<2 \sqrt{q} \\ j \equiv-1(4)}} H\left(j^{2}-4 q\right) j^{h},
$$

provided that $r$ is not too large (a " $H(d)$-calculator" can be found in [7]).

6. Acknowledgments. The author would like to thank the anonymous reviewers for their detailed comments.

\section{REFERENCES}

[1] L. Carlitz, A note on exponential sums, Pacific J. Math., 30 (1969), pp. 35-37.

[2] L. CARLitz, Explicit evaluation of certain exponential sums, Math. Scand., 44 (1979), pp. 5-16.

[3] K. Chinen and T. Hiramatsu, Hyper-Kloosterman sums and their applications to the coding theory, Appl. Algebra Eng. Commun. Comput., 12 (2001), pp. 381-390.

[4] K. Chinen, On some properties of the hyper-Kloosterman codes, Tokyo J. Math., 26 (2003), pp. 55-65.

[5] G. Lachaud And J. Wolfmann, The weights of orthogonals of the extended quadratic binary Goppa codes, IEEE Trans. Inform. Theory, 36 (1990), pp. 686-692.

[6] F. J. MacWilliams and N. J .A. Sloane, The Theory of Error Correcting Codes, Amsterdam: North-Holland, 1977. 
[7] K. Matthews, Some BCMath/PHP number theory programs, Available: http://www.numbertheory.org/php

[8] M. Moisio, A note on evaluations of some exponential sums, Acta Arith., 93 (2000), pp. $117-119$.

[9] M. Moisio, On the number of rational points on some families of Fermat curves over finite fields, Finite Fields Appl., 13 (2007), pp. 546-562.

[10] M. MoIsio, The moments of a Kloosterman sum and the weight distribution of a Zetterberg type binary cyclic code, IEEE Trans. Inform. Theory, 53 (2007), pp. 843-847.

[11] M. MoIsio, On the moments of Kloosterman sums and fibre products of Kloosterman curves, Finite Fields Appl., to appear.

[12] C.J. Moreno And O. Moreno, The MacWilliams-Sloane conjecture on the tightness of the Carlitz-Uchiyama bound and the weights of duals of BCH codes, IEEE Trans. Inform. Theory, 40 (1994), pp. 1894-1907.

[13] V. Pless, Power moment identities on weight distributions in error correcting codes, Information and Control, 6 (1963), pp. 147-152.

[14] R. SChOOF AND M. VAN DER VLUGT, Hecke operators and the weight distributions of certain codes, J. Combin. Theory Ser., A 57 (1991), pp. 163-186. 